\title{
Dose-response effect of an apple extract on postprandial glycaemia is not explained by delayed gastric emptying: a randomised control trial
}

\author{
E. Prpa, W.L. Hall, B. Atkinson, B. Blackstone, E. Leftley, L. Romano, P. Parekh and Y. Wang \\ Diet and Cardiometabolic Health Research Group, Department of Nutritional Sciences, Faculty of Life Sciences \& \\ Medicine, King's College London, 150 Stamford Street, London, SE1 9NH
}

This abstract was awarded the student prize for best oral original communication

Diets with a high glycaemic load may contribute towards an increased risk of developing type 2 diabetes mellitus (T2D). Food and beverages containing large amounts of highly bioaccessible starch and sugars induce pronounced postprandial blood glucose concentrations. Chronic exposure to exaggerated sharp peaks in blood glucose can lead to adverse modifications to functional proteins, oxidative stress and pancreatic beta cell dysfunction. A polyphenol-rich apple extract has been shown to limit the blood glucose excursion following a glucose load ${ }^{(1)}$. Possible mechanisms include inhibition of carbohydrate digestive enzymes activity and/or intestinal glucose transporter activity and/or delaying gastric emptying. Previous research by our group demonstrated that $1.8 \mathrm{~g}$ of apple extract (AE; Appl'In ${ }^{\mathrm{TM}}$ ), containing $1.2 \mathrm{~g}$ apple polyphenols, mixed into a drink consumed before a high starch + sucrose meal, inhibited the incremental area under the curve (iAUC) of postprandial plasma glucose during the early absorption-driven postprandial phase $(0-30 \mathrm{~min})$ by $54 \%$ relative to a placebo drink and meal ${ }^{(2)}$.

Since polyphenol-rich fruit extracts can affect the taste and texture of beverages, the primary aim of the GLU-POMME study was to investigate whether lower doses of $\mathrm{AE}$ also significantly reduced postprandial glycaemia following a starch + sucrose test meal in healthy participants $(\mathrm{N}=30 ; 34 \pm 15 \mathrm{y})$. Four matched test drinks, containing either: 1) $1.8 \mathrm{~g}($ High), 2) $1.35 \mathrm{~g}(\mathrm{Medium})$, or 3$) 0.9 \mathrm{~g}$ (Low) of AE, compared with 4) $0 \mathrm{~g}$ (Placebo), were consumed in random order on separate study visits immediately before the high-carbohydrate test meal (white bread and low polyphenol jam). In a sub-group $(\mathrm{n}=6)$ of participants paracetamol $(1.5 \mathrm{~g})$ was incorporated into drinks to measure gastric emptying rate. Plasma glucose iAUC 0-30 min was significantly lower for each dose of AE v Placebo (mean difference with $95 \%$ CI): High $-15.6 \mathrm{mmol} / \mathrm{L} . \mathrm{min},-23.3,-7.9, P<0.001 ; \mathrm{Medium}-11.3 \mathrm{mmol} / \mathrm{L} . \mathrm{min}$, $-19 \cdot 6,-3 \cdot 0, P<0.002$; Low $-8.99 \mathrm{mmol} / \mathrm{L} / \mathrm{min},-17 \cdot 3,-0 \cdot 7, P<0 \cdot 05$. Serum paracetamol concentrations did not differ between AE drinks and placebo. The paracetamol iAUC 0-30 min for each dose of AE v Placebo (mean difference with $95 \% \mathrm{CI}$ ): High 122.0 mg/L.min, -245.3, 489.3; Medium -23.4 mg/L.min, -296.5, 249.7; Low 79.3 mg/L.min, -183.4, $342 \cdot 0$.

Through this work we have determined that a low dose of AE, $0.9 \mathrm{~g}$, slows the rate of glucose absorption in a broad, healthy population of men and women, with potential applications for new product development in functional foods and beverages. The delaying effect of AE on postprandial glycaemia is not explained by slower gastric emptying, and it is likely that inhibition of carbohydrate digestive enzymes and intestinal glucose transport are the main mechanisms. These results help inform the design of planned in vitro studies to explore the AE polyphenol mechanisms of action.

Financial Support. DIANA Food, Rennes, France.

1. Schulze C, Bangert A, Kottra G et al. (2014) Mol Nutr Food Res 58, 1795-808.

2. Castro-Acosta MJ, Stone S, Mok J et al. (2017) J Nutr Biochem 49, 53-62. 